\title{
LOCAL ENERGY DECAY FOR THE DAMPED PLATE EQUATION IN EXTERIOR DOMAINS
}

\author{
BY \\ SONG JIANG \\ Institut für Angewandte Mathematik der Universität Bonn, Bonn, Germany \\ and Xi'an Jiaotong University, Xi'an, Shaanxi Province, People's Republic of China
}

\begin{abstract}
We establish a rate of local energy decay for solutions of the damped plate equation with variable coefficients in exterior domains by using the spectral analysis to the corresponding stationary problem.
\end{abstract}

1. Introduction. We discuss the local energy decay for solutions of the following damped plate equation (with variable coefficients):

$$
u_{t t}+A u+b u_{t}=0, \quad x \in \Omega, t>0
$$

with boundary and initial conditions

$$
\begin{array}{ccrl}
\left.u\right|_{\partial \Omega} & =0, & & \left.\frac{\partial u}{\partial n}\right|_{\partial \Omega}=0, \\
u(0, \cdot) & =u_{0}, & u_{t}(0, \cdot)=u_{1},
\end{array}
$$

where $u=u(t, x), b=b(x)$ is a real smooth function in $\bar{\Omega}$ and

$$
A u \equiv A(x) u:=\sum_{|\alpha|,|\beta| \leq 2}(-1)^{|\alpha|} \partial_{x}^{\alpha}\left(a_{\alpha \beta}(x) \partial_{x}^{\beta} u\right),
$$

$\Omega \subset \mathbb{R}^{n}$ is an exterior domain with smooth boundary $\partial \Omega, n$ is the exterior normal, $\alpha=\left(\alpha_{1}, \ldots, \alpha_{n}\right)$ and $\beta=\left(\beta_{1}, \ldots, \beta_{n}\right)$ are multi-indices, and for any multi-index $\alpha=\left(\alpha_{1}, \ldots, \alpha_{n}\right), \partial_{x}^{\alpha}=\partial_{1}^{\alpha_{1}} \cdots \partial_{n}^{\alpha_{n}}\left(|\alpha|=\alpha_{1}+\cdots+\alpha_{n}\right), a_{\alpha \beta}$ are real, smooth functions in $\bar{\Omega}$ and subscripts denote partial differentiations.

The study of local energy decay is not only the interest in the decay rates itself but also its importance for the investigation of $L^{p}-L^{q}$ estimates for solutions of (1.1)(1.3) (cf. [11,9]), which plays a very important role in discussing the global existence of smooth solutions to the corresponding nonlinear equations (cf. Klainerman and Ponce [5], Ponce [8], Shibata and Tsutsumi [11] for nonlinear wave and evolution equations).

Received September 6, 1990.

1991 Mathematics Subject Classification. 35B40, 35K35.

Key words and phrases. The damped plate equation, variable coefficients, local energy decay, spectral analysis.

(C)1992 Brown University 
For $A u=\Delta^{2} u$ and $b(x)=1$, Racke [9] in 1989 used a generalized Fourier transform (also called generalized eigenfunction expansion, cf. Leis [6] and Wilcox [15]) and investigated decay estimates for solutions of (1.1)-(1.3) with different boundary conditions from (1.2). For the boundary conditions $\left.u\right|_{\partial \Omega}=\left.\Delta u\right|_{\partial \Omega}=0$, he implicitly gave $L^{1}-L^{\infty}$, resp. $L^{p}-L^{q}$, estimates for $n \geq 3$, resp. $n \geq 5$. For $\partial u /\left.\partial n\right|_{\partial \Omega}=\partial \Delta u /\left.\partial n\right|_{\partial \Omega}=0$ and $n=3$, he also obtained the local energy decay estimate. In [9] the complement of $\Omega$ is assumed to be star-shaped, resp. convex, because the associated kernel of the generalized Fourier transform has to be estimated pointwise.

Recently, the author [4] studied the local energy decay for (1.1)-(1.3) with $A u=$ $\Delta^{2} u$ and $b(x)=1$ by using a method similar to that used by Shibata [10] for damped wave equations (i.e., the spectral analysis to the corresponding stationary problem, also cf. Vainberg [12-14] for hyperbolic systems). There, by virtue of the dissipative term $u_{t}$, the complement of $\Omega$ is not required to be star-shaped or convex.

The purpose of this paper is to investigate the local energy decay for solutions of (1.1)-(1.3). To obtain the desired results for (1.1)-(1.3) the main idea in [4] still works, but we have to employ some different arguments in the proof (i.e., using the cut-off functions, a similar technique to that used by Iwashita and Shibata [3] for hyperbolic systems with variable coefficients), since the equation (1.1) has the variable coefficients. Before stating the main result of this paper, we introduce the following assumptions for (1.1)-(1.3).

Assumption 1.1. (i) $a_{\alpha \beta}(x)=a_{\beta \alpha}(x)$ for $x \in \Omega$ and $|\alpha|,|\beta| \leq 2$,

$$
\sum_{|\alpha|,|\beta| \leq 2}\left(a_{\alpha \beta} \partial_{x}^{\beta} u, \partial_{x}^{\alpha} u\right) \geq \gamma\left\|D_{x}^{2} u\right\|^{2}
$$

for any $u \in C_{0}^{\infty}(\Omega)$ and some constant $\gamma>0$.

(ii) $b(x) \geq b_{0}$ for all $x \in \bar{\Omega}$ and some constant $b_{0}>0$.

(iii) There is a constant $a_{0}>0$ such that for $|x| \geq a_{0}$

$$
A u=\Delta^{2} u, \quad b(x)=1, \quad \text { and } \quad a_{\alpha \beta}(x)=0
$$

for $|\alpha|$ or $|\beta| \leq 1$.

DEFINITION 1.1. We say that $\left(u_{0}, u_{1}\right)$ satisfy the compatibility conditions of order $L-1$ for $L \geq 1$ if

$$
u_{0} \in W^{2 L, 2}(\Omega), \quad u_{1} \in W^{2(L-1), 2}(\Omega)
$$

and

$$
\left.u_{j}\right|_{\partial \Omega}=\left.\frac{\partial u_{j}}{\partial n}\right|_{\partial \Omega}=0 \quad \text { for } 0 \leq j \leq L-1,
$$

where $u_{j}$ is defined for $j \geq 2$ by

$$
u_{j}:=-A u_{j-2}-b u_{j-1}
$$

(formally $u_{j}(\cdot)=\partial_{t}^{j} u(0, \cdot)$ ). The main result of this work is 
THEOREM 1.1 (Local Energy Decay). Let $n \geq 5, r_{0}>0$ be a fixed number with $B_{r_{0}}$ $\supset \mathbb{R}^{n} \backslash \Omega, L \geq 2$ an integer, and $r$ and $r^{\prime}$ any real numbers with $r^{\prime} \geq r \geq$ $\max \left\{r_{0}+3, a_{0}+1\right\}$. Let Assumption 1.1 be satisfied. Assume that $u_{j} \in W^{2(L-j)+\overline{6}, 2}(\Omega)$, $\operatorname{supp} u_{j} \subset \Omega_{r}(j=0,1)$, and $\left(u_{0}, u_{1}\right)$ satisfy the compatibility conditions of order $L-1$. Then there is a constant $C=C\left(N, L, r, r^{\prime}\right)$ such that

$$
\left\|\partial_{t}^{N} u(t)\right\|_{2(L-N)+1,2, \Omega_{r^{\prime}}} \leq C(1+t)^{-(n / 4+N)}\left(\left\|u_{0}\right\|_{2 L+6,2}+\left\|u_{1}\right\|_{2 L+4,2}\right)
$$

for any $0 \leq N \leq L$ and $t \geq 0$, where the constant $a_{0}>0$ is the same as in Assumption 1.1.

REMARK 1.1. The notation that appears above is defined later.

REMARK 1.2. Using the above local energy decay, following the similar arguments of those employed in Sect. 4 of [11] (also cf. [4, 9]), one can obtain the $L^{p}-L^{q}$ estimates for solutions of $(1.1)-(1.3)$.

REMARK 1.3. The restriction on the dimension is just out of a technical difficulty of the method used here. In the estimate of $R_{0}(0) f$ in Sect. 3 (cf. (3.1)) we want to bound the integral $\int_{|\xi| \leq 1}|\xi|^{-4} d \xi$; therefore, we have to assume that $n \geq 5$.

Our proof of Theorem 1.1 is based on a sequence of theorems. Roughly speaking, to show Theorem 1.1, we first study the behaviour of $R^{\prime}(\tau) f$ in $\{\tau \in \mathbb{C}, \operatorname{Im} \tau \leq 0\}$ for $f \in L_{r}^{2}(\Omega)$, where $R^{\prime}(\tau) f$ satisfies $\left(A-\tau^{2}+i \tau b\right) R^{\prime}(\tau) f=f$ in $\Omega$ and $R^{\prime}(\tau) f=$ $\partial R^{\prime}(\tau) f / \partial n=0$ on $\partial \Omega$. Then we show that solutions of (1.1)-(1.3) are equal to $(2 \pi)^{-1} \int R^{\prime}(\tau) f e^{i \tau t} d \tau$ for some $f$ depending on $u_{0}$ and $u_{1}$. We integrate by parts with respect to $\tau$ and utilize the behaviour of $R^{\prime}(\tau) f$ near $\tau=0$ to obtain the desired result.

This paper is organized as follows. Section 2 proves some properties of $R^{\prime}(\tau) f$ for $\tau \in\{\tau \in \mathbb{C}, \operatorname{Im} \tau<0\}$ and for $\tau \in \mathbb{R}^{1},|\tau| \rightarrow \infty$. In Sect. 3 we introduce a $C^{k}$ space of the fractional power derivatives and give some of its properties, by which we investigate the behaviour of $R^{\prime}(\tau) f$ near $\tau=0$ in Sect. 4. Section 5 gives the proof of Theorem 1.1.

Notation. $i=\sqrt{-1}$. For any multi-index $\alpha=\left(\alpha_{1}, \ldots, \alpha_{n}\right)$, we set $\partial_{x}^{\alpha}=$ $\partial_{1}^{\alpha_{1}} \cdots \partial_{n}^{\alpha_{n}},|\alpha|=\alpha_{1}+\cdots+\alpha_{n}$. For any multi-indices $\alpha$ and $\beta, \alpha \geq \beta$ means that $\alpha_{j} \geq \beta_{j}$ for $j=1, \ldots, n$. For a nonnegative integer $N$, we denote

$$
\begin{aligned}
& D_{x}^{N} u=\left(\partial_{x}^{\alpha} u ;|\alpha|=N\right), \quad \bar{D}_{x}^{N} u=\left(\partial_{x}^{\alpha} u ; 0 \leq|\alpha| \leq N\right), \\
& D^{N} u=\left(\partial_{t}^{j} \partial_{x}^{\alpha} u ; j+|\alpha|=N\right), \quad \bar{D}^{N} u=\left(\partial_{t}^{j} \partial_{x}^{\alpha} u ; 0 \leq j+|\alpha| \leq N\right) .
\end{aligned}
$$

Let $G \subset \mathbb{R}^{n}$ be a domain. We denote by $W^{m, p}(G), W_{0}^{m, p}(G)$ the usual Sobolev spaces with the norm $\|\cdot\|_{m, p, G} \cdot W^{0, p}(G)=L^{p}(G)$ with the norm $\|\cdot\|_{p, G}$ (cf. Adams [1]). For simplicity we use the abbreviations:

$$
\begin{aligned}
& \|\cdot\|_{m, p, G}=\|\cdot\|_{m, p} \quad \text { when } G=\Omega \text { or } G=\mathbb{R}^{n} \text { and } \\
& \|\cdot\|_{p, G}=\|\cdot\|_{p} \quad \text { when } G=\Omega \text { or } G=\mathbb{R}^{n} .
\end{aligned}
$$

$\|\cdot\|$ and $(\cdot, \cdot)_{0}$ stand for the norm and the scalar product in $L^{2}(\Omega)$ or $L^{2}\left(\mathbb{R}^{n}\right)$. For Banach spaces $B_{1}$ and $B_{2}, \mathscr{L}\left(B_{1}, B_{2}\right)$ is the Banach space of all bounded linear 
operators from $B_{1}$ to $B_{2}$ with its operator norm $\|\cdot\|_{\mathscr{L}\left(B_{1}, B_{2}\right)}$. In particular, when $B=B_{1}=B_{2}$ we write $\mathscr{L}(B)=\mathscr{L}(B, B)$ and $\|\cdot\|_{\mathscr{L}(B)}=\|\cdot\|_{\mathscr{L}(B, B)}$. For an open set $\omega \in \mathbb{C}, \operatorname{Anal}(\omega ; B) \quad\left(\right.$ resp. $\left.C^{N}(G ; B)\right)$ denote the space of all $B$-valued functions that are holomorphic in $\omega$ (resp. $N$-times continuously differentiable in $G) . C_{0}^{N}(G ; B)=\left\{u \in C^{N}(G ; B)\right.$; $\left.\operatorname{supp} u \Subset G\right\}$. For simplicity, let $C^{N}(G)=$ $C^{N}(G ; \mathbb{C})$ and $C_{0}^{N}(G)=C_{0}^{N}(G ; \mathbb{C})$. Let

$$
H_{\Delta}(G)=\overline{\left\{C_{0}^{\infty}(G),\|\cdot\|_{\Delta, G}\right\}},
$$

where $\|f\|_{\Delta, G}^{2}=\int_{G}\left|D_{x}^{2} f(x)\right|^{2} d x$. For any $r>0$, we put

$$
\begin{gathered}
G_{r}:=\{x \in G ;|x|<r\}, \quad B_{r}:=\left\{x \in \mathbb{R}^{n} ;|x|<r\right\}, \\
L_{r}^{2}(G):=\left\{f \in L^{2}(G), \operatorname{supp} f \subset G_{r}\right\} .
\end{gathered}
$$

Throughout this paper, $r_{0}>0$ (the same as in Theorem 1.1) is a fixed large number with $B_{r_{0}} \supset \mathbb{R}^{n} \backslash \Omega$. Let $\pi(x) \in C^{\infty}\left(\mathbb{R}^{n}\right)$ such that $\pi(x)=1$ for $x \in B_{r_{0}}$ and $\pi(x)=\exp \left(-|x|^{2}\right)$ for $x \in \mathbb{R}^{n} \backslash B_{r_{0}+1}$. We denote $\langle f\rangle_{G}:=\int_{G} \pi(x)|f(x)|^{2} d x$ and define the Banach spaces $\mathscr{J}_{0}$ and $\mathscr{J}_{0}^{\prime}$ by

$$
\begin{aligned}
& \mathscr{J}_{0}:=\left\{f \in L_{\mathrm{loc}}^{2}\left(\bar{\Omega} ;\langle f\rangle_{\Omega}<+\infty\right\},\right. \\
& \mathcal{J}_{0}^{\prime}:=\left\{f \in L_{\mathrm{loc}}^{2}\left(\mathbb{R}^{n}\right) ;\langle f\rangle_{\mathbb{R}^{n}}<+\infty\right\}
\end{aligned}
$$

with norms $\langle\cdot\rangle_{G}$ and $\langle\cdot\rangle_{\mathbb{R}^{n}}$, respectively. Put

$$
\begin{aligned}
& \mathscr{J}_{4}:=\left\{f \in L_{\mathrm{loc}}^{2}(\bar{\Omega}) ;\left\langle\bar{D}_{x}^{4} f\right\rangle_{\Omega}<+\infty,\left.f\right|_{\partial \Omega}=\partial f /\left.\partial n\right|_{\partial \Omega}=0\right\}, \\
& \mathscr{J}_{4}^{\prime}:=\left\{f \in L_{\mathrm{loc}}^{2}\left(\mathbb{R}^{n}\right) ;\left\langle\bar{D}_{x}^{4} f\right\rangle_{\mathbb{R}^{n}}<+\infty\right\} .
\end{aligned}
$$

We also use the following abbreviations:

$$
\|\cdot\|_{\Delta}=\|\cdot\|_{\Delta, G} \quad \text { and }\langle\cdot\rangle_{G}=\langle\cdot\rangle \quad \text { if } G=\Omega \text { or } G=\mathbb{R}^{n} .
$$

We denote by $\hat{f}(\xi)$ the Fourier transform of $f$ and by $\mathscr{F}^{-1}(\hat{f})(x)$ the inverse Fourier transform of $\hat{f}(\xi)$. Finally, throughout this paper, $C$ (sometimes used as $C(a, b, \ldots)$ to emphasize that $C$ depends on $a, b, \ldots)$ denotes various constants.

REMARK 1.4. It is easy to see from Definition 1.1 that

$$
\left\|u_{j}\right\|_{m, p} \leq C\left(\left\|u_{0}\right\|_{2 j+m, p}+\left\|u_{1}\right\|_{2(j-1)+m, p}\right) \text { for } j \geq 2 .
$$

Hence (1.5) guarantees that $u_{j}$ and $\partial u_{j} / \partial n(0 \leq j \leq L-1)$ are well defined on $\partial \Omega$.

EXAMPLE 1.1. Let

$$
A u:=\Delta\left(c_{1}(x) \Delta u\right)-\nabla \cdot\left(c_{2}(x) \nabla u\right)-c_{3}(x) \cdot \nabla u-\operatorname{div}\left(c_{3}(x) u\right)+c_{4}(x) u,
$$

where $c_{1}, c_{2}, c_{3}$, and $c_{4}$ are real, smooth functions in $\bar{\Omega}$ satisfying:

(1) $c_{1}(x)=1, c_{2}(x)=c_{4}(x)=0$, and $c_{3}(x)=0$ for $|x| \geq a_{0}$ and

(2) $c_{1}(x) \geq \gamma, c_{2}(x), c_{4}(x) \geq 0$ for $x \in \Omega$. 
Then, the differential operator defined above satisfies Assumption 1.1.

REMARK 1.5. If we take $c_{1}(x)=b_{1}(x)=1, c_{2}(x)=c_{4}(x)=0$, and $c_{3}(x)=0$ for $x \in \Omega$, then $A u:=\Delta^{2} u$. Hence, Theorem 1.1 extends the results in [4].

2. Definition and some properties of $R^{\prime}(\tau)$ in $\kappa$. We denote

$$
\begin{aligned}
& \kappa_{1}=\left\{\tau \in \mathbb{C} ; \operatorname{Re} \tau \neq 0, \operatorname{Im} \tau<b_{0} / 2\right\}, \\
& \kappa_{2}=\{\tau \in \mathbb{C} ; \operatorname{Re} \tau=0, \operatorname{Im} \tau<0\}, \\
& \kappa=\kappa_{1} \cup \kappa_{2},
\end{aligned}
$$

where the constant $b_{0}>0$ is the same as in Assumption 1.1 in the introduction. We shall make use of the following two lemmas.

Lemma 2.1. Let $u \in H_{\Delta}(\Omega), v \in H_{\Delta}\left(\mathbb{R}^{n}\right)$, and $n \geq 5$. Then there is a constant $C>0$ such that

$$
\int_{\Omega_{r}}|u|^{2} \leq C r^{4}\|u\|_{\Delta}^{2} \quad \text { for } r \geq r_{0}, \quad \int_{B_{r}}|v|^{2} \leq C r^{4}\|v\|_{\Delta}^{2} \quad \text { for } r>0 .
$$

Lemma 2.2. (i) Let $u \in H_{\Delta}(\Omega) \cap W^{2,2}(\Omega), A u \in L^{2}(\Omega)$. Also assume that Assumption 1.1(i) is satisfied. Then $u \in W^{4,2}(\Omega)$ satisfying

$$
\|u\|_{4,2} \leq C(\|A u\|+\|u\|) \text {. }
$$

(ii) Let $f \in L_{r}^{2}(\Omega)$ with $r \geq r_{0} . u \in H_{\Delta}(\Omega)$ satisfies $A u=f$ in $\Omega$. Let Assumption 1.1(i) be satisfied and $a_{\alpha \beta}=0$ for $|x| \geq a_{0}$ and $|\alpha|$ or $|\beta| \leq 1$. Then $D_{x}^{3} u, D_{x}^{4} u \in L^{2}(\Omega)$ and

$$
\left\|D_{x}^{3} u\right\|+\left\|D_{x}^{4} u\right\| \leq C(r)\left\{\|f\|+\|u\|_{\Delta}\right\},
$$

where $a_{0}$ is the same as in Assumption 1.1.

The proof of Lemma 2.1 can be found in [4]. We can show Lemma 2.2 by using the regularity theory of elliptic equations (cf. Theorem 9.8 and the proof of Lemmas 9.2-9.5 in [2]) and here we omit the proof. Now we introduce the operator $R^{\prime}(\tau)$ and study some of its properties.

Theorem 2.3. Let Assumption 1.1(i) be satisfied. Then for all $\tau \in \kappa$, there is a unique, bounded linear operator $R^{\prime}(\tau) f: L^{2}(\Omega) \rightarrow H_{\Delta}(\Omega) \cap W^{4,2}(\Omega)$ such that for $f \in L^{2}(\Omega), R^{\prime}(\tau) f$ satisfies

$$
\left(A-\tau^{2}+i \tau b\right) R^{\prime}(\tau) f=f \quad \text { in } \Omega,
$$

and the following estimates

$$
\left\|D_{x}^{j} R^{\prime}(\tau) f\right\| \leq C \xi_{j}(\tau)\|f\|, \quad j=0, \ldots, 4,
$$


where

$$
\begin{aligned}
& \xi_{0}(\tau)= \begin{cases}\left(|\operatorname{Re} \tau|\left|2 \operatorname{Im} \tau-b_{0}\right|\right)^{-1} & \text { for } \tau \in \kappa_{1}, \\
\left(|\tau|+|\tau|^{2}\right)^{-1} & \text { for } \tau \in \kappa_{2} ;\end{cases} \\
& \xi_{1}(\tau)=\xi_{0}(\tau)+\xi_{2}(\tau) \text { for } \tau \in \kappa ; \\
& \xi_{2}(\tau)= \begin{cases}\left\{\xi_{0}(\tau)\left(1+\xi_{0}(\tau)\left(\left|(\operatorname{Re} \tau)^{2}-(\operatorname{Im} \tau)^{2}\right|+|\operatorname{Im} \tau|\right)\right)\right\}^{1 / 2} & \text { for } \tau \in \kappa_{1}, \\
\left(|\tau|+|\tau|^{2}\right)^{-1 / 2} & \text { for } \tau \in k_{2}\end{cases} \\
& \xi_{3}(\tau)=\xi_{2}(\tau)+\xi_{4}(\tau) \text { for } \tau \in \kappa ; \\
& \xi_{4}(\tau)=\left(1+|\tau|+|\tau|^{2}\right) \xi_{0}(\tau)+1 \text { for } \tau \in \kappa ;
\end{aligned}
$$

and

$$
\left\{\begin{array}{l}
\left\|\bar{D}_{x}^{2} R^{\prime}(\tau) f\right\| \leq C(r)\|f\| \\
\left\|\bar{D}_{x}^{1} D^{3} R^{\prime}(\tau) f\right\| \leq C(r)\left(|\tau|^{2}+|\tau|\right)\|f\|
\end{array}\right.
$$

for any $\tau \in \kappa$ with $|\operatorname{Re} \tau| \leq 1$ and $\operatorname{Im} \tau \leq-2$.

Proof. For any $u \in H_{\Delta}(\Omega) \cap W^{4,2}(\Omega)$, we integrate by parts and use Assumption $1.1(\mathrm{i})$ to infer that

$$
\begin{aligned}
(A u, & u)_{0}-\tau^{2}(u, u)_{0}+i \tau(b u, u)_{0} \\
& =\sum_{|\alpha|,|\beta| \leq 2}\left(a_{\alpha \beta} \partial_{x}^{\beta} u, \partial_{x}^{\alpha} u\right)_{0}-\tau^{2}(u, u)_{0}+i \tau(b u, u)_{0} \\
& \geq \gamma\|u\|_{\Delta}^{2}-\tau^{2}(u, u)_{0}+i \tau(b u, u)_{0} .
\end{aligned}
$$

Using (2.6) and the interpolation inequality (cf. [1])

$$
\left\|D_{x}^{1} u\right\| \leq C\left(\varepsilon_{0}\right)\left(\varepsilon\left\|D_{x}^{2} u\right\|+\varepsilon^{-1}\|u\|\right) \text { for any } 0<\varepsilon \leq \varepsilon_{0}
$$

where $\varepsilon_{0}>0$ is a fixed finite number, we obtain by the generalized Lax-Milgram lemma that for any $\tau \in \kappa$ and $f \in L^{2}(\Omega)$ there is a unique $R^{\prime}(\tau) f \in H_{\Delta}(\Omega) \cap$ $W^{2,2}(\Omega)$ such that $\left(A-\tau^{2}+i \tau\right) R^{\prime}(\tau) f=f$, which combined with Lemma 2.2(i) implies $R^{\prime}(\tau) f \in W^{4,2}(\Omega)$. We thus proved the existence and uniqueness of the operator $R^{\prime}(\tau), \tau \in \kappa$. By virtue of Lemma 2.2, (2.6), (2.7), and the estimate that $\operatorname{Re}\left(-\tau^{2}(u, u)+i \tau(b u, u)\right) \geq 2\|u\|^{2}$ for any $\tau \in \kappa$ with $|\operatorname{Re} \tau| \leq 1$ and $\operatorname{Im} \tau \leq-2$, we obtain (2.3) and (2.5) after a straightforward computation. This completes the proof.

Let $\tau$ and $\tau_{1}$ be any points in $\kappa$. Put

$$
c(\tau)=\sum_{j=0}^{\infty}\left\{\left(\left(-\tau_{1}^{2}+i \tau_{1} b\right)-\left(-\tau^{2}+i \tau b\right)\right) R^{\prime}\left(\tau_{1}\right)\right\}^{j} .
$$

In view of Theorem 2.3 we see that the series on the right-hand side of $(2.8)$ converges in $\mathscr{L}\left(L^{2}(\Omega)\right)$ for $\tau$ close to $\tau_{1}$. Furthermore it is easy to see that for such $\tau$, its limit is indeed the inverse of the operator, $1+\left(\left(-\tau^{2}+i \tau b\right)-\left(-\tau_{1}^{2}+i \tau_{1} b\right)\right) R^{\prime}\left(\tau_{1}\right)$. Hence, for such $\tau$ we have for $f \in L^{2}(\Omega),\left(A-\tau^{2}+i \tau b\right) R^{\prime}\left(\tau_{1}\right) c(\tau) f=f$ in $\Omega$. So it follows from Theorem 2.3 that $R^{\prime}(\tau)=R^{\prime}\left(\tau_{1}\right) c(\tau)$ for such $\tau$. In particular, 
one has $R^{\prime}(\tau) \in \operatorname{Anal}\left(\kappa ; \mathscr{L}\left(L^{2}(\Omega) ; H_{\Delta}(\Omega) \cap W^{4,2}(\Omega)\right)\right)$. If we differentiate equation (2.2) with respect to $\tau$, utilize Theorem 2.3, and (2.7) with $\varepsilon_{0}=\gamma^{-1 / 2}(\gamma>0)$ and $\varepsilon=|\tau|^{-1 / 2} \quad(|\tau|>\gamma)$, we get

THeOREM 2.4. Let $R^{\prime}(\tau)$ be the same as in Theorem 2.3. Then $R^{\prime}(\tau)$ is in $\operatorname{Anal}\left(\kappa ; \mathscr{L}\left(L^{2}(\Omega) ; H_{\Delta}(\Omega) \cap W^{4,2}(\Omega)\right)\right)$ and satisfies

$$
\left\|\partial_{\tau}^{N} D_{x}^{j} R^{\prime}(\tau) f\right\| \leq C(N, \gamma)|\tau|^{-1+j / 2}\|f\|
$$

for any $\gamma>0$, integer $N \geq 0, \tau \in \mathbb{R}^{1}$ with $|\tau|>\gamma$, and $j=0, \ldots, 4$.

3. The space $C^{k}$ and the behaviour of $R_{0}(\tau)$ near $\tau=0$. In order to investigate the behaviour of $R^{\prime}(\tau)$ near $\tau=0$ we make use of the space $C^{k}$ (of the fractional power derivatives). Throughout this section we denote by $B$ a Banach space with norm $|\cdot|_{B}$.

Definition 3.1. Let $N$ be a positive integer and $k=N+\sigma$ with $0<\sigma \leq 1$. Put

$$
C^{k} \equiv C^{k}\left(\mathbb{R}^{1} ; B\right)=\left\{u \in C^{N-1}\left(\mathbb{R}^{1} ; B\right) \cap C^{\infty}\left(\mathbb{R}^{1} \backslash\{0\}, B\right) ;\langle\langle u\rangle\rangle_{k, B}<\infty\right\},
$$

where

$$
\langle\langle u\rangle\rangle_{k, B}=\sum_{j=0}^{N} \int_{\mathbb{R}^{1}}\left|\frac{d^{j} u(\tau)}{d \tau^{j}}\right|_{B} d \tau+\sup _{h \neq 0}|h|^{-\sigma} \int_{\mathbb{R}^{1}}\left|\frac{d^{N} u(\tau+h)}{d \tau^{N}}-\frac{d^{N} u(\tau)}{d \tau^{N}}\right|_{B} d \tau
$$

for $0<\sigma<1$ and

$$
\begin{aligned}
\langle\langle u\rangle\rangle_{k, B}= & \sum_{j=0}^{N} \int_{\mathbb{R}^{1}}\left|\frac{d^{j} u(\tau)}{d \tau^{j}}\right|_{B} d \tau \\
& +\sup _{h \neq 0}|h|^{-1} \int_{\mathbb{R}^{\prime}}\left|\frac{d^{N} u(\tau+2 h)}{d \tau^{N}}-2 \frac{d^{N} u(\tau+h)}{d \tau^{N}}+\frac{d^{N} u(\tau)}{d \tau^{N}}\right|_{B} d \tau
\end{aligned}
$$

for $\sigma=1 . C^{k}$ is a subspace of the usual Besov space $B_{1, \infty}^{k}\left(\mathbb{R}^{1} ; B\right)$ (see Muramatsu [7]). The following theorem provides a sufficient condition to guarantee that $f$ belongs to $C^{k}$, and its proof can be found in Shibata [10].

THEOREM 3.2. Let $N$ be a positive integer and $f \in C^{\infty}\left(\mathbb{R}^{1} \backslash\{0\}, B\right) \cap C_{0}^{N-1}(I, B)$, where $I=(-2,2)$.

(i) Let $k=N+\sigma$ with $0<\sigma<1$. If $f$ satisfies

(a) For any $\tau \in I \backslash\{0\}$,

$$
\begin{aligned}
& \left|\frac{d^{j} f(\tau)}{d \tau^{j}}\right|_{B} \leq C(f) \quad \text { for any integer } j \in[0, N-1] \\
& \left|\frac{d^{N} f(\tau)}{d \tau^{N}}\right|_{B} \leq C(f)|\tau|^{\sigma-1} ; \quad\left|\frac{d^{N+1} f(\tau)}{d \tau^{N+1}}\right|_{B} \leq C(f)|\tau|^{\sigma-2} .
\end{aligned}
$$

Then $f \in C^{k}\left(\mathbb{R}^{1} ; B\right)$ and $f$ satisfies

$$
\langle\langle f\rangle\rangle_{k, B} \leq C(\sigma, N) C(f), \quad|f(\tau)-f(0)|_{B} \leq C(\sigma, N) C(f)|\tau|^{\sigma} .
$$


(ii) Let $k=N+1$. If $f$ satisfies

(a) There is a $f_{0} \in B$ and a $B$-valued function $f_{1}(\tau)$ defined on $I$ such that

$$
\left(d^{N} / d \tau^{N}\right) f(\tau)=f_{0} \log |\tau|+f_{1}(\tau) \quad \text { for } \tau \in I \backslash\{0\} .
$$

(b) For any $\tau \in I \backslash\{0\}$

$$
\begin{aligned}
& \left|\frac{d^{j} f(\tau)}{d \tau^{j}}\right|_{B} \leq C(f) \quad \text { for any integer } j \in[0, N-1],\left|f_{0}\right|_{B} \leq C(f),\left|f_{1}(\tau)\right|_{B} \leq C(f) ; \\
& \left|\frac{d^{N+1} f(\tau)}{d \tau^{N+1}}\right|_{B} \leq C(f)|\tau|^{-1} ; \quad\left|\frac{d^{N+2} f(\tau)}{d \tau^{N+2}}\right|_{B} \leq C(f)|\tau|^{-2} .
\end{aligned}
$$

Then $f \in C^{k}\left(\mathbb{R}^{1} ; B\right)$ satisfies

$$
\langle\langle f\rangle\rangle_{k, B} \leq C(\sigma, N) C(f), \quad|f(\tau)-f(0)|_{B} \leq C(\sigma, N) C(f)|\tau|^{1 / 2} .
$$

We now state the following theorem established by Shibata in [10], which shows the decay rate of the Fourier transform of functions in $C^{k}$.

THEOREM 3.3. Let $N$ be a positive integer and $0<\sigma \leq 1$. Assume $f \in C^{N+\sigma}\left(\mathbb{R}^{1} ; B\right)$. Then

$$
|g(t)|_{B} \leq C(1+|t|)^{-(N+\sigma)}\langle\langle f\rangle\rangle_{N+\sigma, B},
$$

where $g(t)=(2 \pi)^{-1} \int_{-\infty}^{\infty} f(\tau) \exp (i t \tau) d \tau$.

Next we introduce the operator $R_{0}(\tau)$ and study its properties. For $f \in L_{r}^{2}\left(\mathbb{R}^{n}\right)$, $r>0$ we define

$$
R_{0}(\tau) f=(2 \pi)^{-n} \int e^{i x \xi} \hat{f}(\xi)\left(|\xi|^{4}-\tau^{2}+i \tau\right)^{-1} d \xi, \quad \tau \in \mathbb{R}^{1}
$$

By the discussion at the beginning of Sect. 4 in [4] we know that for $f \in L_{r}^{2}\left(\mathbb{R}^{n}\right)$, $R_{0}(\tau) f$ is well defined in $L_{\text {loc }}^{2}\left(\mathbb{R}^{n}\right)$ for any $\tau \in \mathbb{R}^{1}$. Clearly,

$$
\left(\Delta^{2}-\tau^{2}+i \tau\right) R_{0}(\tau) f=f \text { in } \mathbb{R}^{n} .
$$

The following theorem gives the property of $R_{0}(\tau)$ near $\tau=0$ and is proved in [4]. THEOREM 3.4. Denote $I=[-1 / 2,1 / 2] \backslash\{0\}$. Let $n \geq 5$ and $f \in L_{r}^{2}\left(\mathbb{R}^{n}\right)$, and let $\alpha$ be a multi-index with $0 \leq|\alpha| \leq 4$, and $j$ below an integer. Then the following hold.

(i) $R_{0}(\tau) f \in C^{\infty}\left(I, \mathscr{J}_{4}^{\prime}\right)$, where $\mathscr{J}_{4}^{\prime}$ is the same as in Sec. 1 .

(ii) $\lim _{\tau \rightarrow+0} \partial_{\tau}^{j} \partial_{x}^{\alpha} R_{0}(\tau) f=\lim _{\tau \rightarrow-0} \partial_{\tau}^{j} \partial_{x}^{\alpha} R_{0}(\tau) f$ for any $j \in[0,(n+|\alpha|-5) / 4]$.

(iii) $\left\langle\partial_{\tau}^{j} \partial_{x}^{\alpha} R_{0}(\tau) f\right\rangle \leq C(j, \alpha, r)\|f\|$ for any $j \in[0,(n+|\alpha|-5) / 4]$.

(iv) There are $f_{0}, f_{1}(\tau) \in L_{\text {loc }}^{2}\left(\mathbb{R}^{n}\right)$ for $\tau \in I$ such that

$$
\partial_{\tau}^{j} \partial_{x}^{\alpha} R_{0}(\tau) f=f_{0} \log |\tau|+f_{1}(\tau) \text { for } j=(n+|\alpha|) / 4-1,
$$

and the following holds:

$$
\left\langle f_{0}\right\rangle \leq C\|f\|, \quad\left\langle f_{1}(\tau)\right\rangle \leq C\|f\|, \quad \forall \tau \in I .
$$

(v) $\left\langle\partial_{\tau}^{j} \partial_{x}^{\alpha} R_{0}(\tau) f\right\rangle \leq C(j, \alpha, r)|\tau|^{-(j+1)+(|\alpha|+n) / 4}\|f\| \quad$ for any $j>(n+|\alpha|) / 4-1$. 
As a result of Theorems 3.2 and 3.4 , we get

Corollary 3.5. Let $n \geq 5$, $\alpha$ be a multi-index with $0 \leq|\alpha| \leq 4$, and $j$ below an integer. Let $\phi(\tau) \in C_{0}^{\infty}\left(\mathbb{R}^{1}\right)$ satisfy $\phi(\tau)=1$ for $|\tau| \leq 1 / 4$ and $\phi(\tau)=0$ for $|\tau| \geq 1 / 2$. Then for any integer $N \geq 0$ and $f \in L_{r}^{2}\left(\mathbb{R}^{n}\right)$,

$$
\phi(\tau) \tau^{N} \partial_{x}^{\alpha} R_{0}(\tau) f \in C^{(n+|\alpha|) / 4+N}\left(\mathbb{R}^{1} ; \mathscr{J}_{0}^{\prime}\right) .
$$

Furthermore, the following holds.

$$
\begin{aligned}
& \left\langle\left\langle\phi(\tau) \tau^{N} \partial_{x}^{\alpha} R_{0}(\tau) f\right\rangle\right\rangle_{(n+|\alpha|) / 4+N, \mathscr{g}_{0}^{\prime}} \leq C(n, \alpha, N, \phi, r)\|f\| \quad \text { for } \tau \in \mathbb{R}^{1}, \\
& \left\langle\partial_{x}^{\alpha}\left(R_{0}(\tau)-R_{0}(0)\right) f\right\rangle \leq C(\alpha, r)|\tau|^{(1+j) / 4}\|f\|
\end{aligned}
$$

for $\tau \in \mathbb{R}^{1}$ with $|\tau| \leq 1 / 4$ and $n+|\alpha|=4 k+j$ with $0 \leq j \leq 3$ and some $k \geq 1$.

4. Behaviour of $R(\tau)$ near $\tau=0$. In this section we introduce an operator $R(\tau)$ and study its behaviour near $\tau=0$. Throughout this section let Assumption 1.1 be satisfied, $r \geq \max \left\{r_{0}+3, a_{0}+1\right\}$ a fixed number, where $a_{0}$ is the same as in Assumption 1.1, and let $\langle u, v\rangle_{\Delta}$ denote

$$
\langle u, v\rangle_{\Delta}=\left(D_{x}^{2} u, D_{x}^{2} v\right)_{0} .
$$

By Assumption 1.1 and the Sobolev inequality we see that for $u \in H_{\Delta}(\Omega)$,

$$
\gamma\|u\|_{\Delta}^{2} \leq \sum_{|\alpha|,|\beta| \leq 2}\left(a_{\alpha \beta} \partial_{x}^{\beta} u, \partial_{x}^{\alpha} u\right) \leq C\|u\|_{\Delta}^{2} .
$$

So the norm induced by the scalar product $\sum_{|\alpha|,|\beta| \leq 2}\left(a_{\alpha \beta} \partial_{x}^{\beta} u, \partial_{x}^{\alpha} v\right)$ is equivalent to the norm $\|\cdot\|_{\Delta}$. The Riesz representation theorem implies that for $f \in L_{r}^{2}(\Omega)$, there is a unique $u \in H_{\Delta}(\Omega)$ such that

$$
\sum_{|\alpha|,|\beta| \leq 2}\left(a_{\alpha \beta} \partial_{x}^{\beta} u, \partial_{x}^{\alpha} v\right)=(f, v), \quad \forall v \in H_{\Delta}(\Omega),
$$

which in conjunction with Lemma 2.1 and Lemma 2.2(ii) yields

THeOrem 4.1. Let $n \geq 5$. Denote $\left.H_{\Delta}^{4}(\Omega)\right):=\left\{v \in H_{\Delta}(\Omega) ;\left\|D_{x}^{3} v\right\|,\left\|D_{x}^{4} v\right\|<\infty\right\}$. Then there exists a unique operator $R^{\prime}(0) \in \mathscr{L}\left(L_{r}^{2}(\Omega), H_{\Delta}^{4}(\Omega)\right)$ such that for $f \in$ $L_{r}^{2}(\Omega)$,

$$
\begin{aligned}
& A R^{\prime}(0) f=f \text { in } \Omega, \\
& \left\|D_{x}^{\alpha} R^{\prime}(0) f\right\| \leq C(r)\|f\| \quad \text { for }|\alpha|=2,3,4 .
\end{aligned}
$$

Now we introduce the operator $R(\tau)$.

Definition 4.2. Let $R^{\prime}(\tau) \quad(\tau \in \kappa)$ and $R^{\prime}(0)$ be the same as in Theorem 2.3 and Theorem 4.1, respectively. We denote by $R(\tau)$ the operator

$$
R(\tau): L_{r}^{2}(\Omega) \rightarrow \mathscr{J}_{4},
$$

which is obtained from $R^{\prime}(\tau)$ by contracting the domain of definition of $R^{\prime}(\tau)$ according to (4.4) and considering its range in a wider space $\mathscr{J}_{4}$ (cf. Notation). 
We have by Theorem 2.4 that $R(\tau) \in \operatorname{Anal}\left(\kappa ; \mathscr{L}\left(L_{r}^{2}(\Omega), \mathscr{J}_{4}\right)\right)$. In the sequel we study the property of $R(\tau)$ near $\tau=0$. Let $\phi$ and $\psi$ be $C^{\infty}\left(\mathbb{R}^{n}\right)$ functions with

$$
\begin{aligned}
& \phi=1 \text { for }|x| \leq r-1 \text { and } \phi=0 \text { for }|x| \geq r, \\
& \psi=1 \text { for }|x| \geq r-2 \text { and } \psi=0 \text { for }|x| \leq r-3 .
\end{aligned}
$$

Using $\phi$ and $\psi$, we introduce the operators $P(\tau)$ and $Q(\tau)$.

$$
\begin{aligned}
P(\tau) f:= & (1-\phi) R_{0}(\tau)\left(\psi f_{0}\right)+\phi R^{\prime}(0) f \\
Q(\tau) f:=- & \left.-\tau^{2}+i \tau b\right) \phi R^{\prime}(0) f+\Delta\left\{(\Delta \phi) R_{0}(\tau)\left(\psi f_{0}\right)+2 \nabla \phi \cdot \nabla R_{0}(\tau)\left(\psi f_{0}\right)\right\} \\
& +\Delta \phi \Delta R_{0}(\tau)\left(\psi f_{0}\right)+2 \nabla \phi \cdot \nabla \Delta R_{0}(\tau)\left(\psi f_{0}\right) \\
& -\sum_{|\alpha|,|\beta| \leq 2}(-1)^{\alpha}\left\{\sum_{0<l \leq \beta}\left(\begin{array}{c}
\beta \\
l
\end{array}\right) \partial_{x}^{\alpha}\left(a_{\alpha \beta} \partial_{x}^{l} \phi \partial_{x}^{\beta-l} R^{\prime}(0) f\right)\right. \\
& \left.+\sum_{0<l \leq \alpha}\left(\begin{array}{l}
\alpha \\
l
\end{array}\right) \partial_{x}^{l} \phi \partial_{x}^{\alpha-l}\left(a_{\alpha \beta} \partial_{x}^{\beta} R^{\prime}(0) f\right)\right\} .
\end{aligned}
$$

Here $R_{0}(\tau)$ is defined by (3.1), $f_{0}=f$ for $x \in \Omega$ and $f_{0}=0$ for $x \in \mathbb{R}^{n} \backslash \Omega$. We have

LEMMA 4.3. For all $\tau \in[-1 / 4,1 / 4]$, the following hold.

(i) $P(\tau) \in \mathscr{L}\left(L_{r}^{2}(\Omega) ; \mathscr{J}_{4}\right)$.

(ii) $Q(\tau)$ is a compact operator mapping $L_{r}^{2}(\Omega)$ into itself.

(iii) For any $f \in L_{r}^{2}(\Omega),\left(A-\tau^{2}+i \tau b\right) P(\tau) f=(1-Q(\tau)) f$.

Proof. (i) and (ii) follow from Theorems 2.3 and 4.1, Lemma 2.1, Corollary 3.5, and the Sobolev imbedding theorem. To show (iii), we observe that for $x \in \Omega$

$$
\begin{aligned}
& \left(\Delta^{2}-\tau^{2}+i \tau\right)(1-\phi) R_{0}(\tau)\left(\psi f_{0}\right) \\
& =(1-\phi) f-\Delta\left\{(\Delta \phi) R_{0}(\tau)\left(\psi f_{0}\right)+2 \nabla \phi \cdot \nabla R_{0}(\tau)\left(\psi f_{0}\right)\right\} \\
& \quad-\Delta \phi \Delta R_{0}(\tau)\left(\psi f_{0}\right)-2 \nabla \phi \cdot \nabla \Delta R_{0}(\tau)\left(\psi f_{0}\right),
\end{aligned}
$$

which is identified to (recall Assumption 1.1(iii), (4.5), and the fact that $r \geq a_{0}+1$ ):

$$
\left(A-\tau^{2}+i \tau b\right)(1-\phi) R_{0}(\tau)\left(\psi f_{0}\right)=\text { the right-hand side of }(4.8) .
$$

By Theorem 4.1 we find after a calculation that

$$
\begin{aligned}
\left(A-\tau^{2}+i \tau b\right) \phi R^{\prime}(0) f= & \phi f+\left(-\tau^{2}+i \tau b\right) \phi R^{\prime}(0) f \\
& -\sum_{|\alpha|,|\beta| \leq 2}(-1)^{\alpha}\left\{\sum_{0<l \leq \beta}\left(\begin{array}{c}
\beta \\
l
\end{array}\right) \partial_{x}^{\alpha}\left(a_{\alpha \beta} \partial_{x}^{l} \phi \partial_{x}^{\beta-l} R^{\prime}(0) f\right)\right. \\
& \left.\quad+\sum_{0<l \leq \alpha}\left(\begin{array}{c}
\alpha \\
l
\end{array}\right) \partial_{x}^{l} \phi \partial_{x}^{\alpha-l}\left(a_{\alpha \beta} \partial_{x}^{\beta} R^{\prime}(0) f\right)\right\},
\end{aligned}
$$

which adding to (4.9) yields (iii) of the lemma.

The following lemma is proved in [4]. 
LEMMA 4.4. Let $n \geq 5$ and $f \in L_{r}^{2}\left(\mathbb{R}^{n}\right)$. Then $R_{0}(0) f \in H_{\Delta}\left(\mathbb{R}^{n}\right)$ and

$$
\left\|D_{x}^{m} R_{0}(0) f\right\| \leq C(r)\|f\|, \quad m=2,3,4 .
$$

LEMMA 4.5. Let $n \geq 5$ and $H_{\Delta}^{4}(\Omega)$ be the same as in Theorem 4.1. Let $H_{\Delta}^{4}\left(\mathbb{R}^{n}\right):=$ $\left\{u \in H_{\Delta}\left(\mathbb{R}^{n}\right) ;\left\|D_{x}^{3} u\right\|,\left\|D_{x}^{4} u\right\|<\infty\right\}$.

(i) If $u \in H_{\Delta}^{4}(\Omega)$ satisfying $A u=0$ in $\Omega$, then $u=0$.

(ii) If $v \in H_{\Delta}^{4}\left(\mathbb{R}^{n}\right)$ satisfying $\Delta^{2} v=0$ in $\mathbb{R}^{n}$, then $v=0$.

Proof. (i) Let $u_{n} \in C_{0}^{\infty}(\Omega)$ such that $\left\|u_{n}-u\right\|_{\Delta} \rightarrow 0$ as $n \rightarrow \infty$. By partial integration we arrive at

$$
0=\left(A u, u_{n}\right)_{0}=\sum_{|\alpha|,|\beta| \leq 2}\left(a_{\alpha \beta} \partial_{x}^{\beta} u, \partial_{x}^{\alpha} u_{n}\right) .
$$

In view of Assumption 1.1, we infer by taking to the limit that

$$
0=\sum_{|\alpha|,|\beta| \leq 2}\left(a_{\alpha \beta} \partial_{x}^{\beta} u, \partial_{x}^{\alpha} u\right)_{0} \geq a_{0}\|u\|_{\Delta}^{2} .
$$

Therefore $D_{x}^{2} u=0$ in $\Omega$, which together with Lemma 2.1 , implies $u=0$ in $\Omega$. This proves (i). (ii) can be shown in a similar fashion. This completes the proof.

We are now able to show the following lemma, which plays an important role in this section.

LemmA 4.6. Let $n \geq 5$. Then there is a constant $c_{0}>0$ such that $\|1-Q(0)\|_{\mathscr{L}\left(L_{r}^{2}(\Omega)\right)}$ $\geq c_{0}$. Here $Q(\tau)$ is defined by $(4.7)$.

Proof. In terms of the compactness of the operator $Q(0): L_{r}^{2}(\Omega) \rightarrow L_{r}^{2}(\Omega)$ and the well-known Riesz-Schauder theory, it suffices to prove that

$$
\text { if } f \in L_{r}^{2}(\Omega) \text { and }(1-Q(0)) f=0 \text { in } \Omega \text {, then } f=0 \text {. }
$$

To show (4.10), let $f \in L_{r}^{2}(\Omega)$ and $(1-Q(0)) f=0$ in $\Omega$. It follows from Lemma 4.3(iii) that

$$
A P(0) f=0, \quad x \in \Omega .
$$

By virtue of (4.6), Lemma 4.4, and Theorem 4.1, we find that $P(0) f \in H_{\Delta}^{4}(\Omega)$. Thus, (4.11) and Lemma 4.5(i) imply

$$
0=P(0) f=(1-\phi) R_{0}(0)\left(\psi f_{0}\right)+\phi R^{\prime}(0) f, \quad x \in \Omega,
$$

which yields

$$
R^{\prime}(0) f=0, \quad f=A R^{\prime}(0) f=0 \quad \text { for } x \in \Omega_{r-1} .
$$

Let $u=R^{\prime}(0) f$ for $x \in \Omega$ and $u=0$ for $x \in \mathbb{R}^{n} \backslash \Omega$. From Theorem 4.1, (4.5), and (4.13), it follows that $u \in H_{\Delta}^{4}\left(\mathbb{R}^{n}\right)$ and

$$
A u=\psi f_{0} \text { for any } x \in \mathbb{R}^{n} \text {. }
$$

Since $A u=\Delta^{2} u$ for $|x| \geq r-1$ and $u=0$ for $|x| \leq r-1$, the equality (4.14) is equivalent to

$$
\Delta^{2} u=\psi f_{0}, \quad x \in \mathbb{R}^{n},
$$


which combined with Lemma 4.4 results in $u-R_{0}(0)\left(\psi f_{0}\right) \in H_{\Delta}^{4}\left(\mathbb{R}^{n}\right)$ and $\Delta^{2}\left(u-R_{0}(0)\left(\psi f_{0}\right)\right)=0$ in $\mathbb{R}^{n}$. By Lemma $4.5\left(\right.$ ii) we conclude that $R^{\prime}(0) f=$ $R_{0}(0)\left(\psi f_{0}\right)$ for $x \in \Omega$, which in conjunction with (4.12) yields $R_{0}(0)\left(\psi f_{0}\right)=0$ for $x \in \Omega$, from which and the fact that $\Delta^{2} R_{0}(0)\left(\psi f_{0}\right)=\psi f_{0}$ (cf. (3.2)), it follows that $\psi f_{0}=0$ for $x \in \Omega$. This together with (4.5) and (4.13) yields $f=0$. The proof of the lemma is completed.

Putting together (4.7), the results of Corollary 3.5, and Lemma 4.6, we conclude that

LEMMA 4.7. There is a positive constant $\delta<1 / 4$, such that

$$
\|1-Q(\tau)\|_{\mathscr{L}\left(L_{r}^{2}(\Omega)\right)} \geq c_{0} / 2 \text { for } \tau \in[-\delta, \delta]
$$

where $c_{0}$ is the same as in Lemma 4.6 .

Lemma 4.7 guarantees that the inverse operator $(1-Q(\tau))^{-1} \in \mathscr{L}\left(L_{r}^{2}(\Omega)\right)$ exists for $\tau \in[-\delta, \delta]$ and that

$$
\left\|(1-Q(\tau))^{-1}\right\|_{\mathscr{L}\left(L_{r}^{2}(\Omega)\right)} \leq c_{0} / 2 \text { for any } \tau \in[-\delta, \delta],
$$

which in conjunction with Lemma 4.3 gives that for any $f \in L_{r}^{2}(\Omega)$ and $\tau \in[-\delta, \delta]$

$$
\begin{aligned}
& \left(A-\tau^{2}+i \tau b\right) P(\tau)(1-Q(\tau))^{-1} f=f \text { in } \Omega, \\
& P(\tau)(1-Q(\tau))^{-1} \in \mathscr{L}\left(L_{r}^{2}(\Omega) ; \mathscr{J}_{4}\right) .
\end{aligned}
$$

Recalling the definition (3.1) of $R_{0}(\tau) f$, keeping in mind that $\left.|| \xi\right|^{4}-\tau^{2}+i \tau \mid \geq$ $C(\tau)\left(1+|\xi|^{2}\right)^{2}$ for all $\tau \in[-\delta, \delta] \backslash\{0\}$, we have $R_{0}(\tau) \in \mathscr{L}\left(L_{r}^{2}(\Omega) ; W^{4,2}(\Omega)\right)$. Hence, $P(\tau)(1-Q(\tau))^{-1} \in \mathscr{L}\left(L_{r}^{2}(\Omega) ; H_{\Delta}(\Omega) \cap W^{4,2}(\Omega)\right)$ for $\tau \in[-\delta, \delta] \backslash\{0\}$. By Theorem 2.3, $R(\tau)=P(\tau)(1-Q(\tau))^{-1}$ for $\tau \in[-\delta, \delta] \backslash\{0\}$. In the case of $\tau=0$, since $P(0)(1-Q(0))^{-1} f$ fulfills the equation $A P(0)(1-Q(0))^{-1} f=f$ in $\Omega$ and $P(0)(1-Q(0))^{-1} f \in H_{\Delta}^{4}(\Omega)$, it follows from the uniqueness that $R^{\prime}(0) f=$ $P(0)(1-Q(0))^{-1} f$. Thus we have proved

Lemma 4.8. Let $\delta>0$ be the same as in Lemma 4.7. Then $R(\tau)=P(\tau)(1-Q(\tau))^{-1}$ for all $\tau \in[-\delta, \delta]$.

Recalling the definition (4.6) of $P(\tau) f$, combining Lemmas 4.7 and 4.8 , Theorems $3.2,3.4$, and 4.1 , we obtain

THEOREM 4.9. Let $\delta$ be the same as in Lemma 4.7 and let $n \geq 5$. Let $\phi(\tau)$ be a $C_{0}^{\infty}\left(\mathbb{R}^{1}\right)$-function with $\phi(\tau)=1$ for $|\tau| \leq \delta / 4$ and $\phi(\tau)=0$ for $|\tau| \geq \delta / 2$. Then for any integer $N \geq 0$, multi-index $\alpha$ with $0 \leq|\alpha| \leq 4$, and $f \in L_{r}^{2}(\Omega)$, we have $\phi(\tau) \tau^{N} \partial_{x}^{\alpha} R(\tau) f \in C^{n / 4+N}\left(\mathbb{R}^{1} ; \mathscr{J}_{0}\right)$. Moreover,

$$
\left\langle\left\langle\phi(\tau) \tau^{N} \partial_{x}^{\alpha} R(\tau) f\right\rangle\right\rangle_{n / 4+N, \mathscr{Z}_{0}} \leq C(n, N, \phi, \alpha, r)\|f\| .
$$

5. The proof of Theorem 1.1. Throughout this section, $u_{j}(j \geq 0)$ are the same as in the introduction (cf. (1.7)). We first express the solution $u(t, x)$ of $(1.1)-(1.3)$ in terms of $R(\tau)$ (i.e., (5.20)). Let

$$
\psi(t) \in C^{\infty}\left(\mathbb{R}^{1}\right), \quad \psi(t)=1 \quad \text { for } t \leq 1 / 2 \quad \text { and } \quad \psi(t)=0 \quad \text { for } t \geq 1 .
$$


Define $g_{L}(t, x)$ by

$$
g_{L}(t, x)=\left(\partial_{t}^{2}+A(x)+b(x) \partial_{t}\right)\left(\sum_{k=0}^{L+1} u_{k}(x) t^{k} / k !\right) \psi(t) .
$$

By (1.7),

Setting

$$
\begin{aligned}
& \partial_{t}^{k} g_{L}(0, \cdot)=0, \quad 0 \leq k \leq L-1, \\
& \partial_{t}^{L} g_{L}(0, \cdot)=b(\cdot) u_{L+1}-A(\cdot) u_{L} .
\end{aligned}
$$

$$
h_{L}(\tau, x)=\int_{0}^{\infty} e^{-i \tau t} g_{L}(t, x) d t,
$$

we utilize (5.3), (5.4), and (1.8), integrate by parts to arrive at

$$
\left\|\partial_{\tau}^{l} h_{L}(\tau)\right\| \leq C(l)(1+|\tau|)^{-(L+1)}\left(\left\|u_{0}\right\|_{2 L+6,2}+\left\|u_{1}\right\|_{2 L+4,2}\right)
$$

for any $\tau \in \mathbb{C}$ with $\operatorname{Im} \tau \leq 0$ and any $l \geq 0$, and

$$
h_{L} \in \operatorname{Anal}\left(\mathbb{C}_{-} ; L_{r}^{2}(\Omega) \cap C^{\infty}\left(\mathbb{R}^{1}, L_{r}^{2}(\Omega)\right) .\right.
$$

Here $\mathbb{C}_{-}=\{\tau \in \mathbb{C} ; \operatorname{Im} \tau<0\}$. Let

$$
w_{L}(t, \cdot)=\frac{1}{2 \pi} \int_{-\infty}^{\infty} e^{i \tau t} R(\tau) h_{L}(\tau, \cdot) d \tau .
$$

It follows from Theorems 2.4 and 4.9 , the definition (4.4) of $R(\tau)$, and (5.6) that

$$
R(\tau) h_{L}(\tau) \in \operatorname{Anal}\left(\mathbb{C}_{-}, \mathscr{J}_{4}\right) \cap C^{0}\left(\mathbb{R}^{1} ; \mathscr{J}_{4}\right) .
$$

From (2.3)-(2.4) in Theorem 2.3 and (5.5), it follows that

$$
\begin{aligned}
& \left\|R(\tau) h_{L}(\tau)\right\| \leq C(r)|\operatorname{Re} \tau|^{-2}(1+|\tau|)^{-L}\left(\left\|u_{0}\right\|_{2 L+6,2}+\left\|u_{1}\right\|_{2 L+4,2}\right), \\
& \left\|\bar{D}_{x}^{1} D_{x}^{1} R(\tau) h_{L}(\tau)\right\| \leq C(r)|\operatorname{Re} \tau|^{-2}(1+|\tau|)^{-(L-1)}\left(\left\|u_{0}\right\|_{2 L+6,2}+\left\|u_{1}\right\|_{2 L+4,2}\right), \\
& \left\|\bar{D}_{x}^{1} D_{x}^{3} R(\tau) h_{L}(\tau)\right\| \leq C(r)|\operatorname{Re} \tau|^{-2}(1+|\tau|)^{-(L-2)}\left(\left\|u_{0}\right\|_{2 L+6,2}+\left\|u_{1}\right\|_{2 L+4,2}\right),
\end{aligned}
$$

for $\tau \in \mathbb{C}_{\text {- }}$ and $|\operatorname{Re} \tau| \geq 1$. By (5.8) we may move the contour up in the integral (5.7) and obtain by (5.9)-(5.11) that

$$
w_{L}(t, \cdot)=\frac{1}{2 \pi} \int_{-\infty}^{\infty} e^{i(\mu+i \sigma) t} R(\mu+i \sigma) h_{L}(\mu+i \sigma, \cdot) d \mu
$$

for any $\sigma \leq 0$. In particular, letting $\sigma \leq-2$ in (5.12), one gets by (5.5), (5.9)-(5.11), and (2.5) in Theorem 2.3 that

$$
\begin{aligned}
& w_{L} \in C^{L-2}\left((-\infty, \infty) ; \mathscr{J}_{4}\right), \\
& \partial_{t}^{L-1} \bar{D}_{x}^{1} D_{x}^{1} w_{L} \in C^{0}\left((-\infty, \infty) ; L^{2}(\Omega)\right), \\
& \partial_{t}^{L} w_{L} \in C^{0}\left((-\infty, \infty) ; L^{2}(\Omega)\right) .
\end{aligned}
$$

If we use (5.9), (5.12), and (2.5) in Theorem 2.3, we infer

$$
\left\langle w_{L}(t)\right\rangle \leq C(r) e^{-\sigma t}\left(\left\|u_{0}\right\|_{2 L+6,2}+\left\|u_{1}\right\|_{2 L+4,2}\right)
$$


for any $\sigma \leq-2$ and $t<0$. Therefore, $w_{L}(t, x)=0$ for $t<0$ and $x \in \Omega$, which together with (5.13) implies

$$
\begin{aligned}
& \partial_{t}^{k} w_{L}(0, x)=0 \quad \text { for } 0 \leq k \leq L, \\
& \left.w_{L}(t, \cdot)\right|_{\partial \Omega}=\left.\frac{\partial w_{L}(t, \cdot)}{\partial n}\right|_{\partial \Omega}=0, \quad t \geq 0 .
\end{aligned}
$$

Let $\tilde{g}(t, x)=g_{L}(t, x)$ for $t \geq 0$ and $\tilde{g}(t, x)=0$ for $t<0$. By (5.3), $\tilde{g} \in$ $C^{0}\left((-\infty, \infty), L_{r}^{2}(\Omega)\right)$. Moreover, one has

$$
\frac{1}{2 \pi} \int_{0}^{\infty} \int_{-\infty+i \sigma}^{\infty+i \sigma} e^{i \tau(t-s)} d \tau g_{L}(s, \cdot) d s=\tilde{g}(t, \cdot)=g_{L}(t, \cdot) \quad \text { for } t \geq 0, \sigma<0
$$

We use (5.4), (5.12), and (5.17) to arrive at

$$
\begin{array}{ll}
w_{L}(t, \cdot)=\frac{1}{2 \pi} \int_{-\infty+i \sigma}^{\infty+i \sigma} e^{i \tau t} R(\tau) h_{L}(\tau, \cdot) d \tau, & \sigma<0, \\
\left(\partial_{t}^{2}+A(x)+b(x) \partial_{t}\right) w_{L}(t, x)=g_{L}(t, x), & t \geq 0, x \in \Omega .
\end{array}
$$

Performing the usual $L^{2}$ energy method, we have by (5.15)-(5.16) and (5.19) that

$$
u(t, x)=\left(\sum_{k=0}^{L+1} u_{k}(x) t^{k} / k !\right) \psi(t)-w_{L}(t, x) .
$$

We have to estimate $w_{L}(t, x)$ in order to complete the proof of Theorem 1.1. Let $\rho(\tau) \in C_{0}^{\infty}\left(\mathbb{R}^{1}\right)$ such that $\rho(\tau)=1$ if $|\tau| \leq \delta / 4$ and $=0$ if $|\tau| \geq \delta / 2$, where $\delta>0$ is the same number as in Theorem 4.9. Denote

$$
w_{L}^{1}(t, \cdot)=(1 / 2 \pi) \int_{-\infty}^{\infty} e^{i \tau t} \rho(\tau) R(\tau) h_{L}(\tau, \cdot) d \tau, \quad w_{L}^{2}(t, \cdot)=w_{L}(t, \cdot)-w_{L}^{1}(t, \cdot) .
$$

We use (5.5) and Theorems 3.3 and 4.9 to obtain

$$
\begin{aligned}
\left\langle\bar{D}_{x}^{4} \partial_{t}^{N} w_{L}^{1}(t)\right\rangle & \leq C(r, N)(1+|t|)^{-(n / 4+N)}\left\langle\left\langle\tau^{N} \rho(\tau) \bar{D}_{x}^{4} R(\tau) h_{L}(\tau)\right\rangle\right\rangle_{n / 4+N, \mathscr{Z}_{0}} \\
& \leq C(r, N)(1+|t|)^{-(n / 4+N)}\left(\left\|u_{0}\right\|_{2 L+6,2}+\left\|u_{1}\right\|_{2 L+4,2}\right) .
\end{aligned}
$$

On the other hand, we apply the following equality, which follows from (5.7) and partial integration

$$
w_{L}^{2}(t, \cdot)=\frac{1}{2 \pi(i t)^{M}} \int_{-\infty}^{\infty} e^{i \tau t} \frac{d^{M}}{d \tau^{M}}\left((1-\rho(\tau)) R(\tau) h_{K}(\tau)\right) d \tau, \quad \forall M \geq 0,
$$

Theorem 2.4, and (5.5) to see that for any $M \geq 0$

$$
\begin{gathered}
\left\langle\partial_{t}^{N} \bar{D}_{x}^{1} w_{L}^{2}(t)\right\rangle \leq C(M, N, r)(1+|t|)^{-M}\left(\left\|u_{0}\right\|_{2 L+6,2}+\left\|u_{1}\right\|_{2 L+4,2}\right), \quad 0 \leq N \leq L, \\
\left\langle\partial_{t}^{N} \bar{D}_{x}^{1} D_{x}^{2} w_{L}^{2}(t)\right\rangle \leq C(M, N, r)(1+|t|)^{-M}\left(\left\|u_{0}\right\|_{2 L+6,2}+\left\|u_{1}\right\|_{2 L+4,2}\right) \\
0 \leq N \leq L-1 \\
\left\langle\partial_{t}^{N} D_{x}^{4} w_{L}^{2}(t)\right\rangle \leq C(M, N, r)(1+|t|)^{-M}\left(\left\|u_{0}\right\|_{2 L+6,2}+\left\|u_{1}\right\|_{2 L+4,2}\right) \\
0 \leq N \leq L-2 .
\end{gathered}
$$


Therefore, (5.20) together with (5.22) and (5.23) imply that for any $r, r^{\prime} \geq$ $\max \left\{r_{0}+3, a_{0}+1\right\}$ and $t \geq 0$ (here $a_{0}$ is the same number as in Assumption $1.1)$,

$$
\begin{gathered}
\left\|\partial_{t}^{N} \bar{D}_{x}^{1} u(t)\right\|_{2, \Omega_{r^{\prime}}} \leq C\left(N, r, r^{\prime}\right)(1+t)^{-(n / 4+N)}\left(\left\|u_{0}\right\|_{2 L+6,2}+\left\|u_{1}\right\|_{2 L+4,2}\right), \\
0 \leq N \leq L, \\
\left\|\partial_{t}^{N} \bar{D}_{x}^{1} D_{x}^{2} u(t)\right\|_{2, \Omega_{r^{\prime}}} \leq C\left(N, r, r^{\prime}\right)(1+t)^{-(n / 4+N)}\left(\left\|u_{0}\right\|_{2 L+6,2}+\left\|u_{1}\right\|_{2 L+4,2}\right), \\
0 \leq N \leq L-1, \\
\left\|\partial_{t}^{N} D_{x}^{4} u(t)\right\|_{2, \Omega_{r^{\prime}}} \leq C\left(N, r, r^{\prime}\right)(1+t)^{-(n / 4+N)}\left(\left\|u_{0}\right\|_{2 L+6,2}+\left\|u_{1}\right\|_{2 L+4,2}\right), \\
0 \leq N \leq L-2 .
\end{gathered}
$$

To estimate higher-order derivatives of $u$, we need the following a priori estimate for the elliptic operator $A$; its proof may be found in [2].

LemMA 5.1. Let $r_{1}, r_{2}$ be any positive numbers with $r_{1}>r_{2} \geq r_{0}$, and $g \in$ $W^{m+4,2}\left(\Omega_{r_{1}}\right),\left.g\right|_{\partial \Omega}=\left.(\partial g / \partial n)\right|_{\partial \Omega}=0$. Then

$$
\|g\|_{m+4,2, \Omega_{r_{2}}} \leq C\left(r_{1}, r_{2}\right)\left(\|A g\|_{m, 2, \Omega_{r_{1}}}+\|g\|_{2,2, \Omega_{r_{1}}}\right) .
$$

Now differentiation of (1.1) with respect to $t$ gives

$$
A \partial_{t}^{N-2} u=-b \partial_{t}^{N-1} u-\partial_{t}^{N} u \quad \text { in } \Omega, \quad 2 \leq N \leq L .
$$

Applying Lemma 5.1 to (5.25), using (5.24) and remembering that $\left(u_{0}, u_{1}\right)$ satisfy the compatibility conditions of order $L-1$, we can obtain by induction that

$$
\left\|\partial_{t}^{N} u(t)\right\|_{2(L-N)+2, \Omega_{r^{\prime}}} \leq C\left(N, L, r, r^{\prime}\right)(1+t)^{-(n / 4+N)}\left(\left\|u_{0}\right\|_{2 L+6,2}+\left\|u_{1}\right\|_{2 L+4,2}\right)
$$

for $0 \leq N \leq L$, any $r, r^{\prime} \geq \max \left\{r_{0}+3, a_{0}+1\right\}$, and $t \geq 0$. This completes the proof of Theorem 1.1.

Acknowledgment. This work was supported by the Sonderforschungsbereich 256 of the Deutsche Forschungsgemeinschaft at the University of Bonn.

\section{REFERENCES}

[1] R. A. Adams, Sobolev Spaces, Academic Press, New York, 1975

[2] S. Agmon, Lectures on Elliptic Boundary Value Problems, Van Nostrand, Princeton, NJ, 1965

[3] H. Iwashita and Y. Shibata, On the analyticity of spectral functions for some exterior boundary value problems, Glasnik Math. 23, 291-313 (1988)

[4] S. Jiang, $L^{p}-L^{q}$ estimates for solutions to the damped plate equation in exterior domains, Results in Math. 18, 231-253 (1990)

[5] S. Klainerman and G. Ponce, Global, small amplitude solutions to nonlinear evolution equations, Comm. Pure Appl. Math. 36, 133-141 (1983)

[6] R. Leis, Initial Boundary Value Problems in Mathematical Physics, Teubner, Stuttgart, and Wiley, Chichester, 1986

[7] T. Muramatsu, On Besov spaces and Sobolev spaces of generalized functions defined in a general region, Publ. Res. Inst. Math. Sci. 9, 325-396 (1977)

[8] G. Ponce, Global existence of small solutions to a class of nonlinear evolution equations, Nonlinear Anal. 9, 399-418 (1985) 
[9] R. Racke, Decay rates for solutions of damped systems and generalized Fourier transforms, J. Reine Angew. Math. 412, 1-19 (1990)

[10] Y. Shibata, On the global existence of classical solutions of second order fully nonlinear hyperbolic equations with first order dissipation in the exterior domain, Tsukuba J. Math. 7, 1-68 (1983)

[11] Y. Shibata and Y. Tsutsumi, On a global existence theorem of small amplitude solutions for nonlinear wave equations in an exterior domain, Math. Z. 191, 165-199 (1986)

[12] B. R. Vainberg, On the analytical properties of the resolvent for a certain class of operator-pencils, Math. USSR-Sb. 6, 241-273 (1968)

[13] B. R. Vainberg, On exterior elliptic problems polynomially depending on a spectral parameter, and the asymptotic behaviour for large time of solutions of nonstationary problems, Math. USSR-Sb. 21, 221-239 (1973)

[14] B. R. Vainberg, On the short wave asymptotic behaviour of solutions of stationary problems and the asymptotic behaviour as $t \rightarrow \infty$ of solutions of non-stationary problems, Russian Math. Surveys 30, 1-58 (1975)

[15] C. H. Wilcox, Scattering theory for the d'Alembert equation in exterior domains, Lecture Notes in Math., vol. 442, Springer-Verlag, Berlin, 1975 